\title{
Bone marrow mesenchymal stem cell- derived exosomes promote plasminogen activator inhibitor 1 expression in vascular cells in the local microenvironment during rabbit osteonecrosis of the femoral head
}

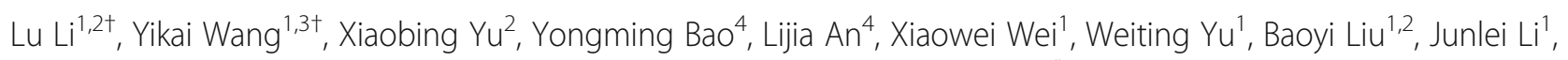
Jiahui Yang ${ }^{1}$, Yan Xia ${ }^{5}$, Ge Liu', Fang Cao ${ }^{1}$, Xiuzhi Zhang ${ }^{1}$ and Dewei Zhao ${ }^{1,2^{*}}$ (D

\begin{abstract}
Background: Nontraumatic osteonecrosis of the femoral head (NONFH) is a highly disabling orthopedic disease in young individuals. Plasminogen activator inhibitor 1 (PAI-1) has been reported to be positively associated with NONFH. We aimed to investigate the dysregulating PAI-1 in bone marrow mesenchymal stem cells (BMMSCs) and vascular cells in rabbit steroid-induced NONFH.

Methods: To verify the hypothesis that BMMSCs could promote thrombus formation in a paracrine manner, we collected exosomes from glucocorticoid-treated BMMSCS (GB-Exo) to determine their regulatory effects on vascular cells. microRNA sequencing was conducted to find potential regulators in GB-Exo. Utilizing gain-of-function and knockdown approaches, we testified the regulatory effect of microRNA in exosomes.

Results: The expression of PAl-1 was significantly increased in the local microenvironment of the femoral head in the ONFH model. GB-Exo promoted PAl-1 expression in vascular smooth muscle cells and vascular endothelial cells. We also revealed that miR-451-5p in GB-Exo plays a crucial role for the elevated PAl-1. Moreover, we identified miR133b-3p and tested its role as a potential inhibitor of PAl-1.

Conclusions: This study provided considerable evidence for BMMSC exosomal miR-mediated upregulation of the fibrinolytic regulator PAI-1 in vascular cells. The disruption of coagulation and low fibrinolysis in the femoral head will eventually lead to a disturbance in the microcirculation of NONFH. We believe that our findings could be of great significance for guiding clinical trials in the future.
\end{abstract}

Keywords: Nontraumatic osteonecrosis of the femoral head, PAl-1, Bone marrow mesenchymal stem cells, Exosome, miRNA

\footnotetext{
* Correspondence: zhaodewei2016@163.com

${ }^{\dagger} \mathrm{Lu}$ Li and Yikai Wang contributed equally to this work.

${ }^{1}$ National-Local Joint Engineering Laboratory for the Development of

Orthopedic Implant Materials, Affiliated Zhongshan Hospital of Dalian

University, Dalian, Liaoning, People's Republic of China

${ }^{2}$ Department of Orthopedics, Affiliated Zhongshan Hospital of Dalian

University, Dalian, Liaoning, People's Republic of China

Full list of author information is available at the end of the article
}

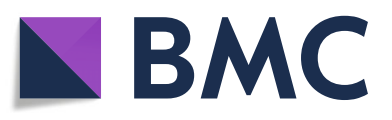

( ) The Author(s). 2020 Open Access This article is licensed under a Creative Commons Attribution 4.0 International License, which permits use, sharing, adaptation, distribution and reproduction in any medium or format, as long as you give appropriate credit to the original author(s) and the source, provide a link to the Creative Commons licence, and indicate if changes were made. The images or other third party material in this article are included in the article's Creative Commons licence, unless indicated otherwise in a credit line to the material. If material is not included in the article's Creative Commons licence and your intended use is not permitted by statutory regulation or exceeds the permitted use, you will need to obtain permission directly from the copyright holder. To view a copy of this licence, visit http://creativecommons.org/licenses/by/4.0/. The Creative Commons Public Domain Dedication waiver (http://creativecommons.org/publicdomain/zero/1.0/) applies to the data made available in this article, unless otherwise stated in a credit line to the data. 


\section{Background}

Nontraumatic osteonecrosis of the femoral head (NONFH or nontraumatic-ONFH) is a chronic and destructive disease in orthopedics with high disability rates. Glucocorticoid (GC) usage is one of the main risk factors [1]. GC-induced NONFH is assumed to cause thrombosis to occur in bone blood vessels, leading to a sequential process of blood flow obstruction, venous high pressure, arterial ischemic, osseous hypoxia, loss of bone integrity, and collapse of the subchondral bone $[2,3]$. Plasminogen activator inhibitor 1 (PAI-1) can suppress the conversion of plasminogen to plasmin and reduce the levels of fibrinolysis in blood vessels, leading to the formation of thrombus [4, 5]. Numerous studies have established a strong association between abnormal PAI-1 levels or activity and a wide range of diseases. Most PAI-1-related human diseases are caused by elevated antigenic concentrations and their inhibitory activity, which are associated with venous thrombosis [4, 6, 7].

Accumulating evidence has indicated the associations between NONFH and PAI-1, and most of them have been found to be a consequence of increased or aberrant PAI-1 in patients with NONFH $[8,9]$. The most reasonable pathomechanism is that increased PAI-1 reduces fibrinolytic function, affects microcirculation in the femoral head, leads to femoral head blood flow reduction, and eventually causes ischemic ONFH $[10,11]$. Upregulated PAI-1 was only identified in the serum of patients with NONFH, but not in osteoarthritis, rheumatoid arthritis, and fracture patients, and so it has the potential to be a diagnostic marker for NONFH [12]. Although steroid treatment can promote plasma PAI-1 levels in the entire body or in a specific type of cells, there are few systematic and conclusive studies about the mechanism of PAI-1 regulation disorder in the femoral head microenvironment of NONFH.

In recent years, stem cell treatments, such as the use of bone marrow mesenchymal stem cells (BMMSCs) to treat ONFH, have achieved good results in both animal models and clinical trials [13, 14]. However, the basic mechanism of BMMSC transplantation treatment remains incompletely understood. Exosomes have gradually attracted attention because they can function in cell-to-cell signaling and can release internal regulatory factors from BMMSCs into the extracellular space, thereby influencing processes in surrounding recipient cells [15]. Growing evidence supports the ideas that exosomes have an important role in different diseases and physiological changes $[16,17]$. At present, recent studies have shown that exosomes mediate regenerative functions in ONFH and other ischemic diseases [18-20]. It has been reported that the release and function of exosomes can be regulated and controlled by glucocorticoids [21]. Moreover, Al-Nedawi et al. demonstrated that exosomes from perivascular cells, such as mast cells, have a significant activating effect on PAI-1 expression in endothelial cells [22]. However, in the pathomechanism of steroid-induced ONFH, whether BMMSC-derived exosomes can interfere with the local blood circulation of the femoral head has not been reported.

Selective removal of some miRNAs in exosomes has also been suggested to be a rapid way of regulating gene expression during the progression of ONFH [23]. Most miRNAs have only been analyzed intracellularly, and few investigations have focused on the role of miRNAs in the extracellular environment. BMMSC miRNAs that are secreted into the extracellular environment could act as key modulators for surrounding tissue cells. Thus, the role of miRNA-mediated cross-talk between BMMSCs and vascular tissues in steroid-induced ONFH remains elusive.

In our previous work, we have successfully constructed a well-validated model of steroid-induced ONFH in rabbit [24], and we verified the preventive effect of ONFH with a combination of an anticoagulant and a vasodilator in systemic administration [25]. But we still have no idea about local microcirculation disorder in the femoral head. Consequently, in this study, we hypothesized that BMMSC-derived exosomes mediate impaired fibrinolytic activity in the early stage of ONFH, and upregulated PAI-1 induce thrombosis in the local femoral head vascular microenvironment. The purpose of this study was to unveil the role of PAI-1 in GC-induced ischemic ONFH in vitro and in vivo. We also revealed that exosomes derived from BMMSCs could be secreted into the extracellular environment to regulate PAI-1 expression in vascular cells. Gene sequencing analysis would provide us with clues for exploring the pathomechanism and identifying potential solutions for hypofibrinolysis in ONFH.

\section{Materials and methods Animals}

All animal care and experimental procedures were approved by the Ethical Committee for Animal Research of Affiliated Zhongshan Hospital of Dalian University. Male 3-month-old healthy adult New Zealand white rabbits weighing $3-4 \mathrm{~kg}$ were purchased from Dalian Medical University, Liaoning, China. The rabbits were kept in specialized animal centers at the Affiliated Zhongshan Hospital of Dalian University and were acclimated for 4 days before initiation of studies.

\section{Animal model and grouping}

NONFH rabbit models were generated by previously well-validated protocols [26]. All rabbits were first 
randomly divided into 2 groups. The rabbits in the experimental (GC) group $(n=8)$ were intravenously injected with $10 \mu \mathrm{g} / \mathrm{kg}$ lipopolysaccharide (Sigma, USA). After $24 \mathrm{~h}$, the experimental (GC) group was intramuscularly injected with $20 \mathrm{mg} / \mathrm{kg}$ of methylprednisolone acetate (Pfizer Manufacturing Belgium, USA) 3 times every $24 \mathrm{~h}$ : the control group $(n=8)$ and the normal group, which was given the same volumes of saline. miR-451-5p group $(n=3)$ : random three of the control group rabbits received AgomiR-451-5p $(25 \mathrm{nmol}$, Genepharma Co., Lt., China) at a dose of $1 \mathrm{~mL}$ by intravenous injection one week after first injection of saline. miR-133b-3p group $(n=3)$ : random three of the experimental (GC) group rabbits received agomiR-133b-3p (25 nmol, Genepharma Co., Lt., China) at a dose of 1 $\mathrm{mL}$ by intravenous injection 1week after the first injection of methylprednisolone acetate during modeling procedure. All rabbits were sacrificed 4 weeks later, and the organs and bone tissue of the lower extremities of the rabbits in the four groups were obtained. For protein extraction, tissue samples were homogenized in liquid nitrogen and then dissolved in icecold protein buffer. For RNA isolation, tissue samples were snap-frozen in liquid nitrogen.

\section{Cell isolation and culture}

BMMSCs were isolated from the femoral head of healthy rabbits by bone marrow aspiration as described [27]. The cells were cultured in Dulbecco's modified Eagle's medium/Ham's F12 nutrient medium (DME/F12, Hyclone, GE, USA) with $10 \%$ fetal bovine serum (FBS, HyClone; GE, USA) and 1\% antibiotic antimycotic solution and changed every 2 days. Vascular endothelial cells (VECs) and vascular smooth muscle cells (VSMCs) were isolated from rabbit thoracic aortas by digestion with different enzymes as described [28, 29]. VECs were maintained in endothelial cell medium (ECM, ScienCell Research Laboratories, USA) and cultured in culture flasks that were coated with gelatin, and the medium was changed every 1-2 days. VSMCs were cultured in the same medium as what was used for BMMSCs, and the medium was changed every day. The cells were separately identified by flow cytometry and immunohistochemistry. All cells were incubated at $37{ }^{\circ} \mathrm{C}$ with $5 \%$ $\mathrm{CO}_{2}$. Cells from passages 3-5 were used in experiments.

\section{Characterization of rabbit cells}

BMMSCs and VECs were identified by flow cytometry. Details on the experimental procedures are provided in Supplementary material. Characterization of VSMCs was performed by alpha-smooth muscle actin $(\alpha-S M A)$-labeled VSMC immunohistochemistry analysis. Detailed procedures are also provided in the supplementary material.

\section{Micro-CT assay}

Micro-CT (Siemens, Inveon Micro-CT, Berlin, Germany) images were obtained to evaluate trabecular bone structure of the femoral head. The scanning protocol was $80 \mathrm{kV}$ and $500 \mu \mathrm{A}$, with an effective pixel size of $15.48 \mu \mathrm{m}$. Based on the CT images, a volume of interest (VOI) was selected from these regions for threedimensional reconstruction and values of bone volume/ total volume (BV/TV), bone surface area/bone volume, trabecular thickness (Tb.Th, $\mathrm{mm}$ ), and trabecular spacing (Tb.Sp, mm) were compared.

\section{Histological and immunohistochemistry (IHC) analysis}

The expression of PAI-1 was observed by IHC analysis. Paraffin sections were dewaxed by routine methods, and antigens were retrieved. After that, $10 \%$ bovine serum albumin (BSA, HyClone; GE, USA) was incubated with the slides in a humid box at room temperature for $60 \mathrm{~min}$, and then primary rabbit anti-PAI-1 antibody (1: 500; Abcam, USA) was incubated with the slides overnight at $4{ }^{\circ} \mathrm{C}$. After washing three times in PBS, the sections were incubated with poly-HRP goat anti-rabbit IgG at $37^{\circ} \mathrm{C}$ for $30 \mathrm{~min}$. Then, the sections were stained with a DAB dye solution and restained with hematoxylin. In addition, hematoxylin and eosin (H\&E) staining and Masson's trichrome staining were conducted to evaluate the histological morphology. The sections were observed under an optical microscope. The number of empty lacunae in five random regions in each section (5 sections in each group) was counted, and the percentage of empty lacunae was defined as the ratio of empty lacuna number to the total lacuna count.

\section{Alkaline phosphatase (ALP) staining}

After 3 days of glucocorticoid stimulation, cell culture medium was removed; then, cells were determined by ALP kits (Beyotime Biotechnology, China) according to the manufacturer's user guide. All cells were observed under a microscope.

\section{Oil red $O$ staining}

After 3 days of glucocorticoid stimulation, cell culture medium was removed. Cells were washed with PBS and fixed with $4 \%$ paraformaldehyde solution at room temperature for $30 \mathrm{~min}$. Then, the freshly prepared Oil Red O working solution (Sangon Biotech, China) was added into culture flask and incubated with the cells at room temperature for $60 \mathrm{~min}$. The staining solution was removed and cells were washed with PBS 3 times. All cells were observed under a microscope.

\section{Isolation and purification of exosome}

BMMSCs were seeded at $5 \times 10^{5}$ cells per $25 \mathrm{~cm}^{2}$ culture flask in $5 \mathrm{~mL}$ of culture medium and were reached 
confluence 5 days later. The cells were cultured in medium supplemented with $10 \%$ serum without FBSderived exosomes and methylprednisolone $(0$ or $5 \mu \mathrm{g} /$ $\mathrm{mL}$ ). Over the next $72 \mathrm{~h}$, the culture medium was collected. The exosomes were collected from the $40 \mathrm{~mL}$ of medium and were subsequently purified via an exosome concentration kit (Liaoning Rengen Biosciences, China) according to the manufacturer's information. The samples were stored at $-80{ }^{\circ} \mathrm{C}$.

\section{Co-culturing BMMSC exosomes with vascular cells}

VECs and VSMCs were seeded at a density of $10^{5}$ cells/ well in a 24-well plate in a 1-mL culture medium before co-culture treatment. After overnight plating, cells were treated with BMMSCs (GC treated or not) exosome for $72 \mathrm{~h}$. The same dose of exosomes $(20 \mu \mathrm{L})$ was added to each group.

\section{Nanoparticle tracking analysis (NTA)}

Particle sizes of exosomes were measured by dynamic light scattering (DLS) (Zetaview, Particle Metrix, Germany).

\section{Transmission electron microscope (TEM)}

Exosomes were dropped on a copper grid for $3 \mathrm{~min}$. Excess liquid was removed with filter paper and dried at room temperature. Next, 3\% ammonium molybdate negative staining solution (Solarbio Science \& Technology, China) was added to the copper grid for $3 \mathrm{~min}$. After removing excess staining solution with filter paper, the grid was examined, and TEM images were recorded (JEM-2100, JEOL, Japan).

\section{Enzyme-linked immunosorbent assay (ELISA)}

The concentration of PAI-1 protein was determined by rabbit PAI-1 ELISA kit (Shanghai Lengton Biotechnology, China) according to the manufacturer's instructions.

\section{Quantitative real-time polymerase chain reaction (qRT- PCR)}

All cells were lysed with RNAiso Plus (Takara Biomedical Technology, Japan) and reverse transcribed using a PrimeScript $^{\text {tix }}$ RT reagent kit with gDNA Eraser (Takara Biomedical Technology, Japan) following the manufacturer's instructions. The generated cDNA was amplified using TB Green ${ }^{\mathrm{Tm}}$ Premix Ex Taq ${ }^{\mathrm{Tm}} I \mathrm{II}$ (Takara Biomedical Technology, Japan). The primers (SERPINE1, ocu-miR133b-3p, ocu-miR-451-5p, U6, and GAPDH) (see Table S1 in the online-only supplementary material) were synthesized by TAKARA BIO (Takara Biomedical Technology, Japan). Target mRNA expression levels were normalized to GAPDH, using the $2^{-\Delta \Delta C t}$ method, and the analysis results are presented as fold change.

\section{Western blotting}

Western blotting was conducted as described [30]. Details on the western blotting experimental procedures are provided in supplementary material. All the antibodies mentioned were as follows: PAI-1 (1:1000) (OmnimAbs, USA), GAPDH (1:1000) (Novusbio, USA).

\section{MicroRNA sequencing}

Extracting and purifying total RNA from cell supernatants was performed via SeraMir ${ }^{\mathrm{Tw}}$ exosome RNA amplification kit (System Biosciences, USA). The purified total RNA was analyzed via Novogene's genomics platform via illumina HiSeq ${ }^{\mathrm{Tu}} 2500 / \mathrm{MiSeq}$ for gene clustering and sequencing. Other detailed procedures are provided in the supplementary material.

\section{Transfection of miR-133b-3p mimics and inhibitor} miR-133b-3p mimics (AgomiR), inhibitor (AntagomiR), stable negative control, and inhibitor N.C. were synthesized by Genepharma Co., Lt. (China) (Table S2). They were transfected into vascular endothelial cells at a final concentration of $40 \mathrm{nM}$ using lipofectamine 2000 (Invitrogen, USA) according to the manufacturer's instructions. All cells were collected for qRT-PCR and western blotting $48 \mathrm{~h}$ after transfection.

\section{Statistical analyses}

Every experiment was repeated at least three times. All data was analyzed by using SPSS version 23.0 (IBM Corp., Armonk, USA). Measurement data were expressed as the mean \pm standard error of mean (s.e.m). Comparisons between groups were analyzed by $t$ tests or ANOVA. A $P$ value of $<0.05$ was considered statistically significant.

\section{Results}

Elevation of PAI-1 expression in GC-induced rabbit ONFH in vivo

Firstly, the experimental (GC) group rabbits $(n=5)$ all showed typical signs of early stage of ONFH. Micro-CT results demonstrated the trabecular bone appeared thinner and sparser. The trabecular space was larger in the subchondral bone in the experimental (GC) group (Fig. 1a). Compared with control group, the mean rate value of $\mathrm{BV} / \mathrm{TV}$ and $\mathrm{Tb}$.Th in experimental group were all significantly lower $(p<0.05$ and $p<0.01)$, whereas the Tb.Sp was significantly higher $(p<0.01)$ (Fig. 1b). On the histologic evaluation, clear differences were observed between the two groups. From Fig. 1c, it can be clearly seen that hypertrophic adipocytes had degenerated accompanied by hyperplasia, even becoming necrotic in the experimental group. Thrombophilia/hypofibrinolysis state was noticed in the Masson staining images. After treatment with glucocorticoid and endotoxin, perivascular fibrosis and neointima formation can be found in the 


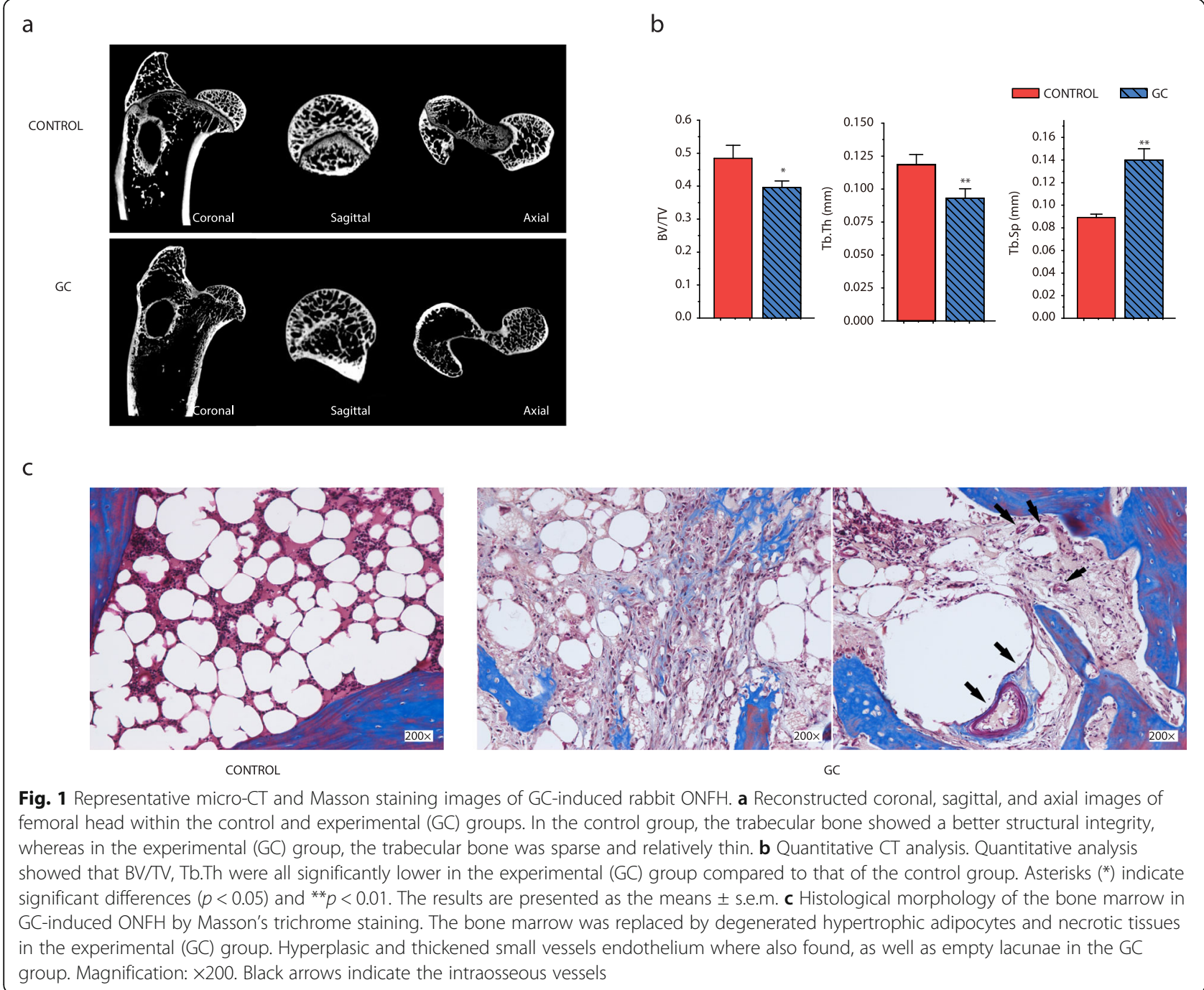

experimental group, as well as hyperplasic and thickened small vessel endothelium. Moreover, most bone lacunae are empty. The micro-CT and staining results indicated the success of the rabbit ONFH models.

To investigate PAI-1 expression in major organ tissues and weight-bearing joints in the lower limbs of GCinduced ONFH rabbit models, we extracted and examined the following tissue samples: major organs (liver, kidney, and lung), hip joint bone (femoral head), knee joint bone (distal femur, proximal tibia, and fibula), ankle joint bone (distal tibia and fibula, talus), and peripheral blood samples (Fig. 2a). First, the expression of PAI-1 mRNA in all experimental groups was significantly higher than it was in the normal control groups $(p<0.01)$. There was a greater increase in the mRNA expression in the liver than there was in the other organs (Fig. 2b). In joint bone tissues, the PAI-1 expression in the femoral head of the experimental group exhibited the greatest increase, which was very similar to that of the blood samples. It is worth noting that the relative mRNA expression in the femoral head region was markedly higher than it was in the other joints (Fig. 2c). Moreover, with a fixed amount of total protein, we found that the PAI-1 concentration in the femoral head and blood were significantly higher than that of the corresponding normal control group $(p<$ 0.01 ). However, we noticed that before and after modeling, the PAI-1 concentrations in the ankle and knee groups showed no significant difference $(p>$ $0.05)$ due to their inherent low abundance (Fig. 2d). Further verifying the change of PAI-1 expression in the femoral head region, the immunohistochemical results showed that the PAI-1 in the local microenvironment of the femoral head in the experimental group was notably higher than it was in the normal control group (Fig. 2e). 

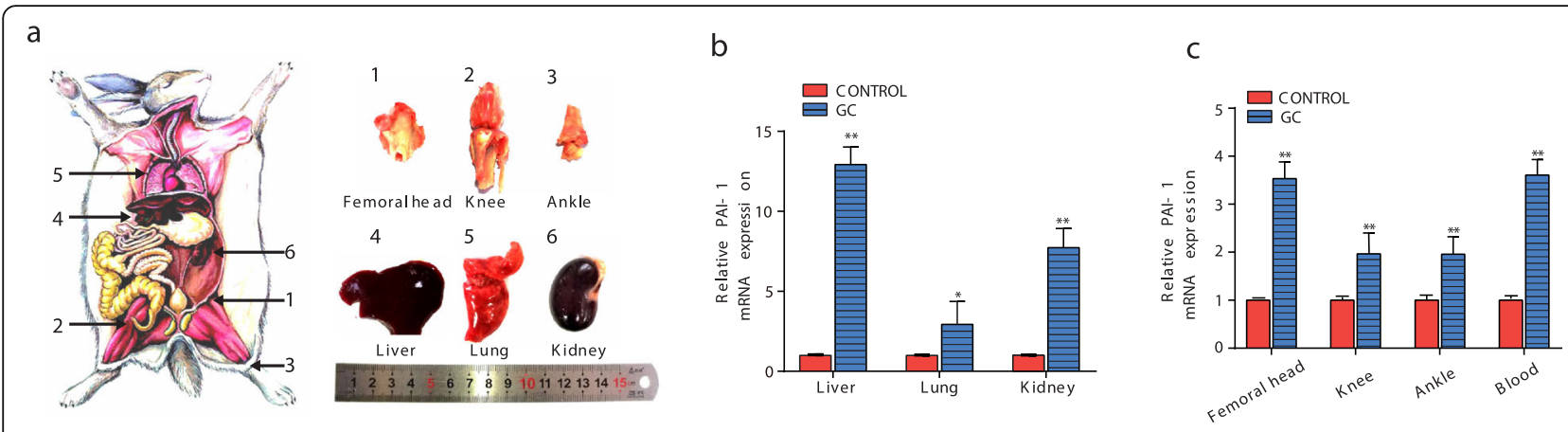

d

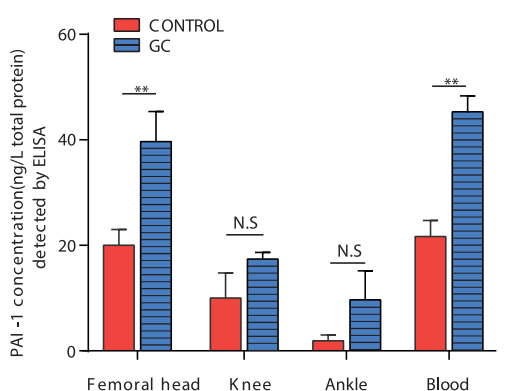

e
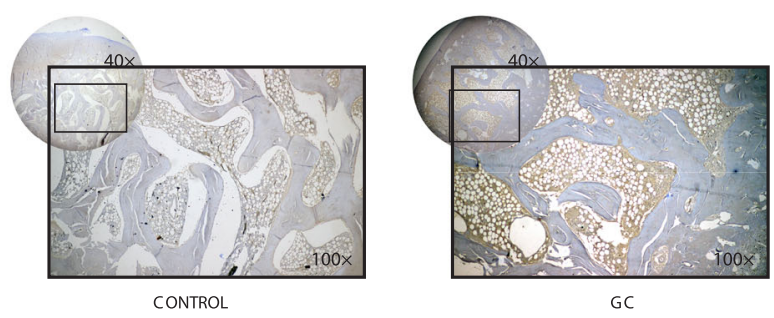

Fig. 2 Elevation of PAl-1 in GC-induced rabbit ONFH in vivo. a Organ and bone tissue of the lower extremities in rabbit were tested $(n=5)$. $\mathbf{b}$ Expression of PAl-1 of organs was significantly increased following GC treatment, as shown by qRT-PCR. c Bone tissue from the lower extremities and blood samples presented a significant increase of PAl-1 expression following GC treatment, as shown by qRT-PCR. $\mathbf{d}$ PAI-1 concentrations in the femoral head, knee, ankle, and blood samples were remarkably increased after the GC treatment, as shown by ELISA. e Representative immunohistochemical staining of PAI-1 in samples of the femoral head from the control and GC-induced experimental groups. Magnification: $\times 40$. Enlarged images shown in the lower right corner at $\times 100$. Asterisks $\left(^{*}\right)$ indicate significant differences $(p<0.05)$ and ${ }^{* *} p<0.01$. The results are presented as the means \pm s.e.m

\section{Characteristics of BMMSCs under GC stimulation and the expression of PAI-1 in vitro}

BMMSCs were isolated from the femoral bone marrow cavity of healthy adult rabbits. Figure $3 \mathrm{a}$ and $\mathrm{b}$ show visualization of spindle-shaped BMMSCs adherently cultured and BMMSC-specific cell surface antigens (positive for CD90 and CD44 and negative for CD45 and CD34). However, after BMMSCs were stimulated with $5 \mu \mathrm{g} / \mathrm{mL}$ methylprednisolone for 3 days, the morphological differences were not observed between the experimental group and the normal control. We did not find changes in surface antigenic features after GC stimulation (Fig. 3b). Moreover, there were no increased numbers of lipid droplets found in GC-treated BMMSCs by oil red $\mathrm{O}$ staining. The ALP staining results also showed that there was no obvious difference between the two groups. These results indicated that BMMSCs did not display a tendency of differentiation into adipocytes or osteoblasts, and they maintained their BMMSC characteristics at our prescribed methylprednisolone concentration and duration time (Fig. 3c). To illustrate the effect of combined methylprednisolone and endotoxin treatment on PAI-1 expression in BMMSCs, the in vivo doses were calculated and adopted for in vitro experiments. Then, $5 \mu \mathrm{g} / \mathrm{mL}$ methylprednisolone, 100 $\mathrm{ng} / \mathrm{mL}$ endotoxin, or both combined doses were added into BMMSC culture medium. PAI-1 expression in BMMSCs was significantly upregulated in all three groups after the drug treatments $(p<0.05)$, but there was no significant difference among the three groups, indicating that there was no synergistic effect between the two drugs (Fig. 3d). Both qRT-PCR and western blotting results displayed the same trend of significantly increased PAI-1 expression $(p<0.05)$ as glucocorticoid concentration increased. However, no statistical differences were found among concentration gradients (Fig. 3e).

\section{Characterization of exosomes from glucocorticoid- stimulated BMMSCs}

Since BMMSCs did not show a differentiation trend, we tried to determine whether they affected other cells with their paracrine exosomes. To fully characterize rabbit BMMSC-derived exosomes, we used exosome-free serum to culture BMMSCs for 3 days under different conditions. First, NTA showed that the particle size distribution of the isolated vesicles was between 30 and $150 \mathrm{~nm}$, and more than $99 \%$ of the microvesicles had a particle size of $130 \mathrm{~nm}$ (Fig. 4a), which is consistent with previous reports of exosomal particle size distribution. 


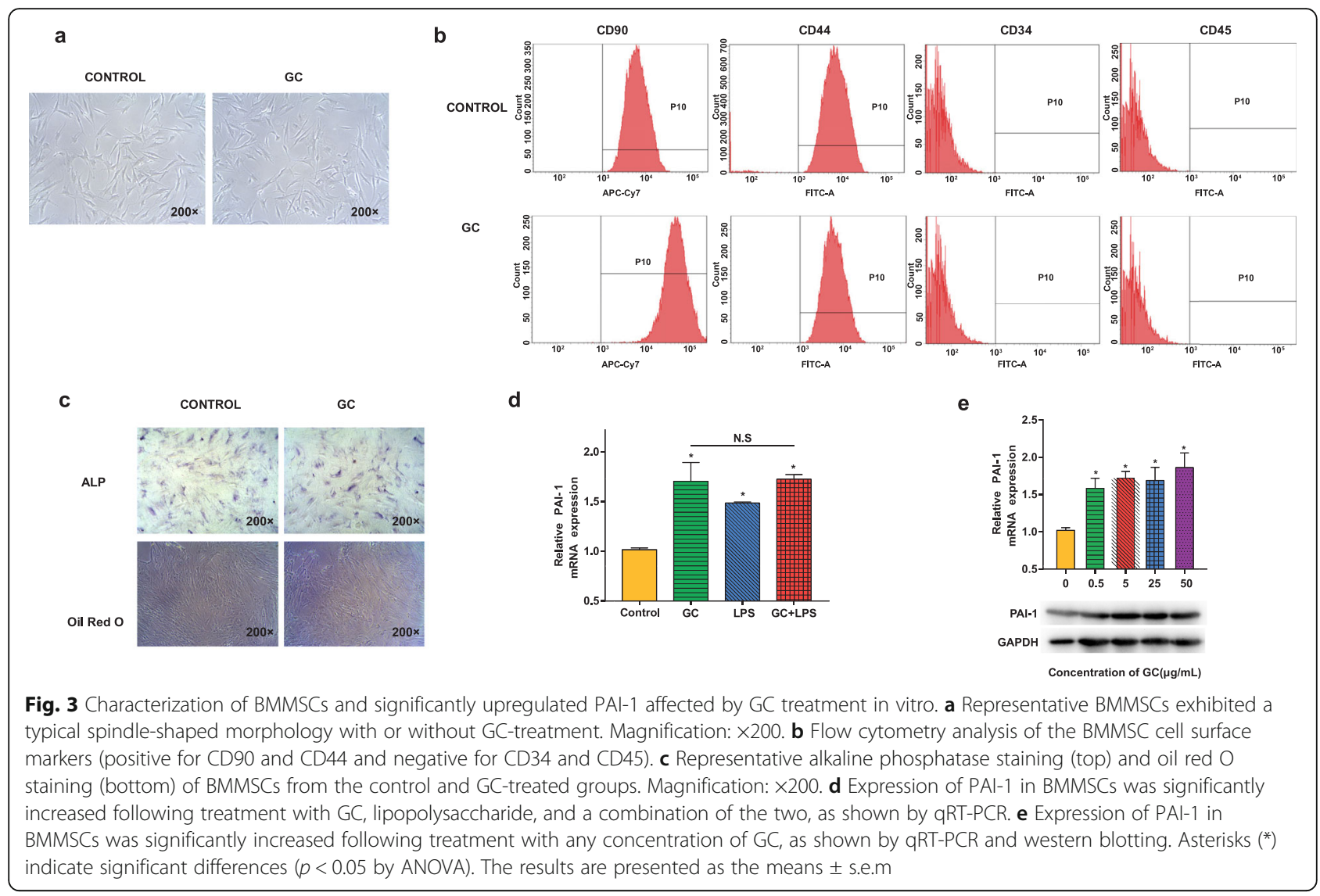

TEM provided us with visual evidence that the typical circular membrane-bound vesicle structure with a size of approximately $100 \mathrm{~nm}$ (Fig. 4b). Western blotting results confirmed the exosomal characteristics by analysis of surface antigen markers; signals were positive for CD9 and HSP70 in the model and control groups (Fig. 4c). For subsequent experiments, we then collected exosomes derived from GC-treated and untreated normal BMMSCs.

\section{BMMSC exosomes regulated PAI-1 expression in VECs and VMSCs}

To explore whether BMMSCs could induce intravascular fibrinolytic disorders through paracrine effects after GC stimulation and whether BMMSC exosomes could regulate the expression of PAI-1, we conducted a series of experiments in primary VMSCs and VECs from New Zealand white rabbits. As shown in Fig. 5a, isolated rabbit primary VMSCs were identified by their

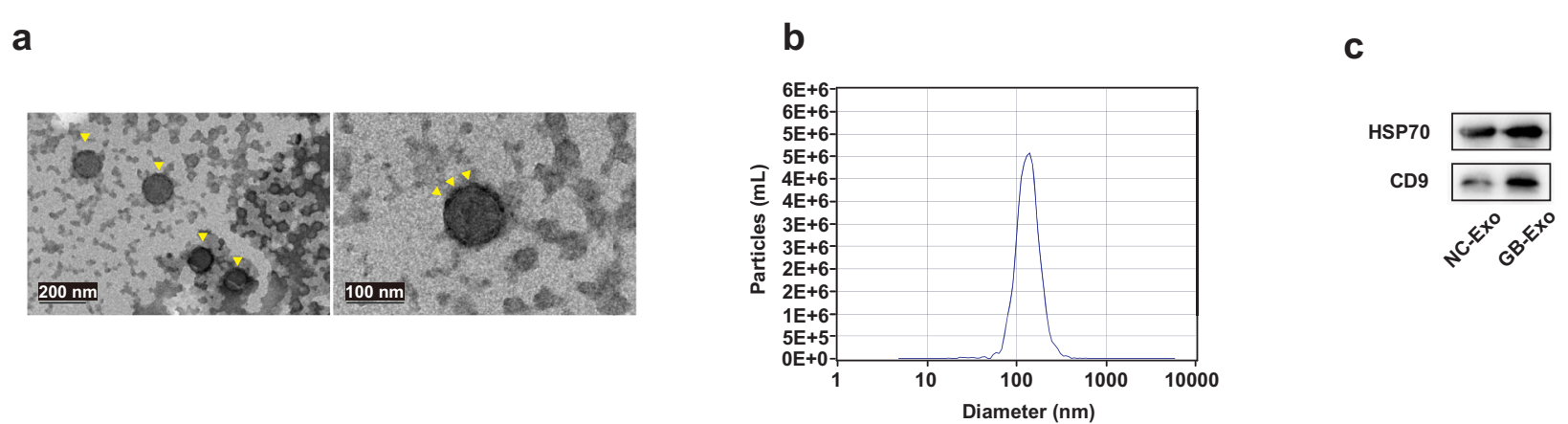

Fig. 4 Characterization of exosomes derived from BMMSCs. a Morphology of exosomes examined by TEM. Arrow heads indicate apparent exosome. Scale bars, $100 \mathrm{~nm}$ and $200 \mathrm{~nm}$. b Particle size distribution of exosomes measured by DLS. c Western blotting analysis of the surface biomarkers CD9 and heat shock proteins HSP70 


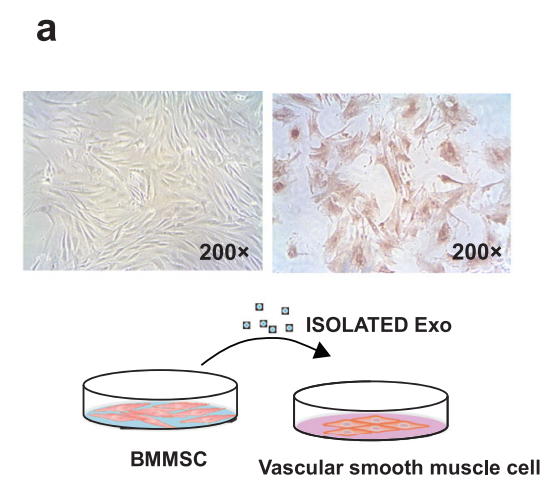

d
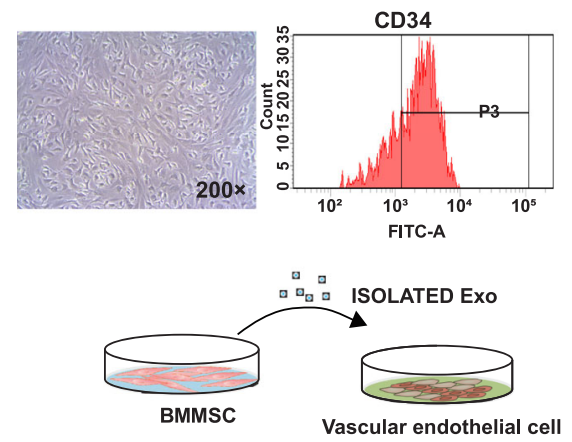

b

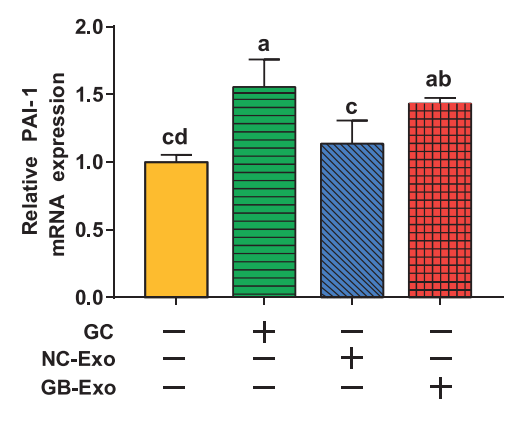

e

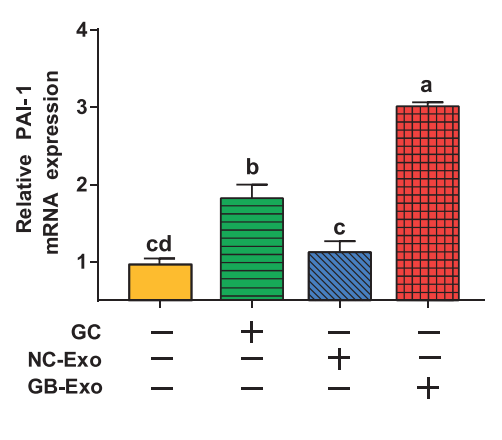

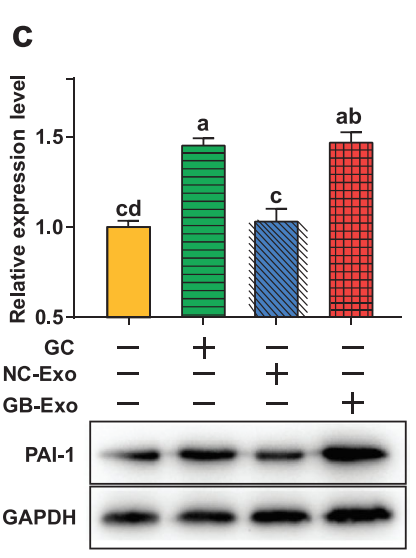

f

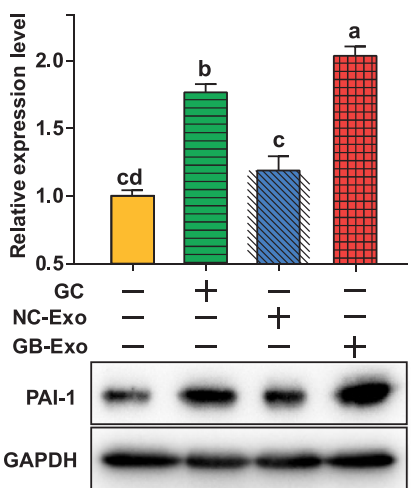

Fig. 5 Exosomes derived from BMMSCs with GC treatment activated VSMCs and VECs to secrete PAI-1. a Representative VSMCs exhibited a typical spindle-shaped morphology (top left). Representative a-SMA immunohistochemical staining of VSMCs (top right). Magnification: $\times 200$. Schematic diagram of the experimental design (bottom). $\mathbf{b}$ Expression of PAl-1 in VSMCs was increased by GC treatment and by GB-Exo treatment, as shown by qRT-PCR and $\mathbf{c}$ western blotting results. $\mathbf{d}$ Representative VEC morphology (top left). Flow cytometry analysis of the cell surface markers on VECS (positive for CD34) (top right). Schematic diagram of the experimental design (bottom). e qRT-PCR and $\mathbf{f}$ western blotting also demonstrated that overexpressed PAl-1 was detected in GC-treated and GB-Exo groups in VECs. In particular, GB-Exo groups exhibited dramatically upregulated PAI-1 expression in VECs. Values in each column with different letters present significant differences $(p<0.05$ by ANOVA). The results are presented as the means \pm s.e.m

morphology under a microscope $(\times 200)$ and by the $\alpha-$ SMA-labeled immunohistochemical results. In the schematic diagram (Fig. 5a), GC-treated BMMSC exosomes (GB-Exo) and normal control BMMSC exosomes (NCExo) were cocultured with VMSCs. First, we found that PAI-1 expression in VMSCs was significantly upregulated by GC treatment as demonstrated in BMMSCs. NC-Exo did not significantly promote the expression of PAI-1 in VMSCs. However, as we assumed, GB-Exo significantly upregulated PAI-1 expression, which was in contrast to the result of the control group. Moreover, there was no significant difference between the GB-Exo group and the GC-treated group (Fig. 5b, c).

Primary rabbit VECs were identified based on the results of morphological microscopic examination and flow cytometry results (positive for CD34) (Fig. 5d). Similar to the above experimental design for VECs, after GC treatment, VECs also upregulated PAI-1 expression, and NC-Exo still did not increase PAI-1 expression. Intriguingly, we found that PAI-1 expression in the GBExo group was significantly higher than it was in the control group and GC-treated group (Fig. 5e and f), which means BMMSCs have a stronger effect on the expression of PAI-1 in VECs than what was achieved following the direct stimulation by GC in vitro. These data suggested that exosomes of BMMSCs have a notable effect on the promotion of PAI-1 in vascular cells.

\section{Exosomal miRNA from BMMSCs regulates PAI-1 expression in VECs}

To understand the properties of BMMSC exosomes for their regulation of PAI-1 in VECs and VMSCs, we examined the content of GB-Exos. Since microRNAs were recently shown to be the main regulators enriched in exosomes that were important for cell-cell communication, we performed microRNA sequencing from GB- 
Exos and NC-Exos. Differentially expressed genes sequencing (DEGseq) analysis provided some clues as to the dysregulated miRNAs induced by GC treatment. The DEGseq results showed that 332 exosomal miRNAs were significantly differentially expressed between the two groups, of which 211 were coexpressed miRNAs, 56 were expressed only in NC-Exos, and 65 were expressed only in GB-Exos (Fig. 6a). In a volcano plot of global miRNA expression (Fig. 6b), an overexpressed microRNA (miR-451) was identified in the right cluster. Relative expression of miR-451-5p in exosomes was also confirmed by qRT-PCR (Fig. 6c). We believe that miR451 might be responsible for the elevated PAI-1 expression in vascular cells. miR-451 has been reported to suppress the PI3K/AKT pathway in various cells [31, 32], and the PI3K/AKT pathway can negatively regulate the expression of PAI-1 [33, 34], resulting in miR-451 indirectly promoting PAI-1 expression.

To find a miRNA that can directly bind to PAI- 1 to induce its downregulation, we screened multiple miRNAs that matched the PAI-1 gene (SERPINE1) in the sequencing results. Excitingly, miR-133b-3p was the only miRNA that was negatively correlated with the expression of target genes. miR-133-3p was significantly downregulated in GB-Exos (Fig. 6b and c). The secondary structure of the miR-133b-3p precursor is shown in Fig. 6d. Using the microRNA target scanning algorithm, we predicted the potential binding site of miR-133b-3p to the target gene (mRNA-XM_002722823 encoded by SERPINE1) (Fig. 6e). To further determine whether miR-133b-3p could downregulate PAI-1 levels, miR-133b-3p inhibitors (antago$\mathrm{miR})$, mimics (agomiR), and negative controls (miR-NC) were transfected into VECs. Our qRT-PCR and western blotting results showed that PAI-1 was significantly downregulated in the miR-133b-3p mimics group. In contrast, the inhibitors increased PAI-1 expression in vitro (Fig. $6 f$ and $\mathrm{g}$ ). Thus, these results supported the idea that miR133b-3p have the potential to restore PAI-1 dysregulation caused by GC treatment.

\section{Effects of miR-451-5p and miR-133b-3p on GC-induced ONFH in vivo}

In order to testify the impact of miR-451-5p and miR$133 \mathrm{~b}-3 \mathrm{p}$ on the rabbit model of GC-induced ONFH

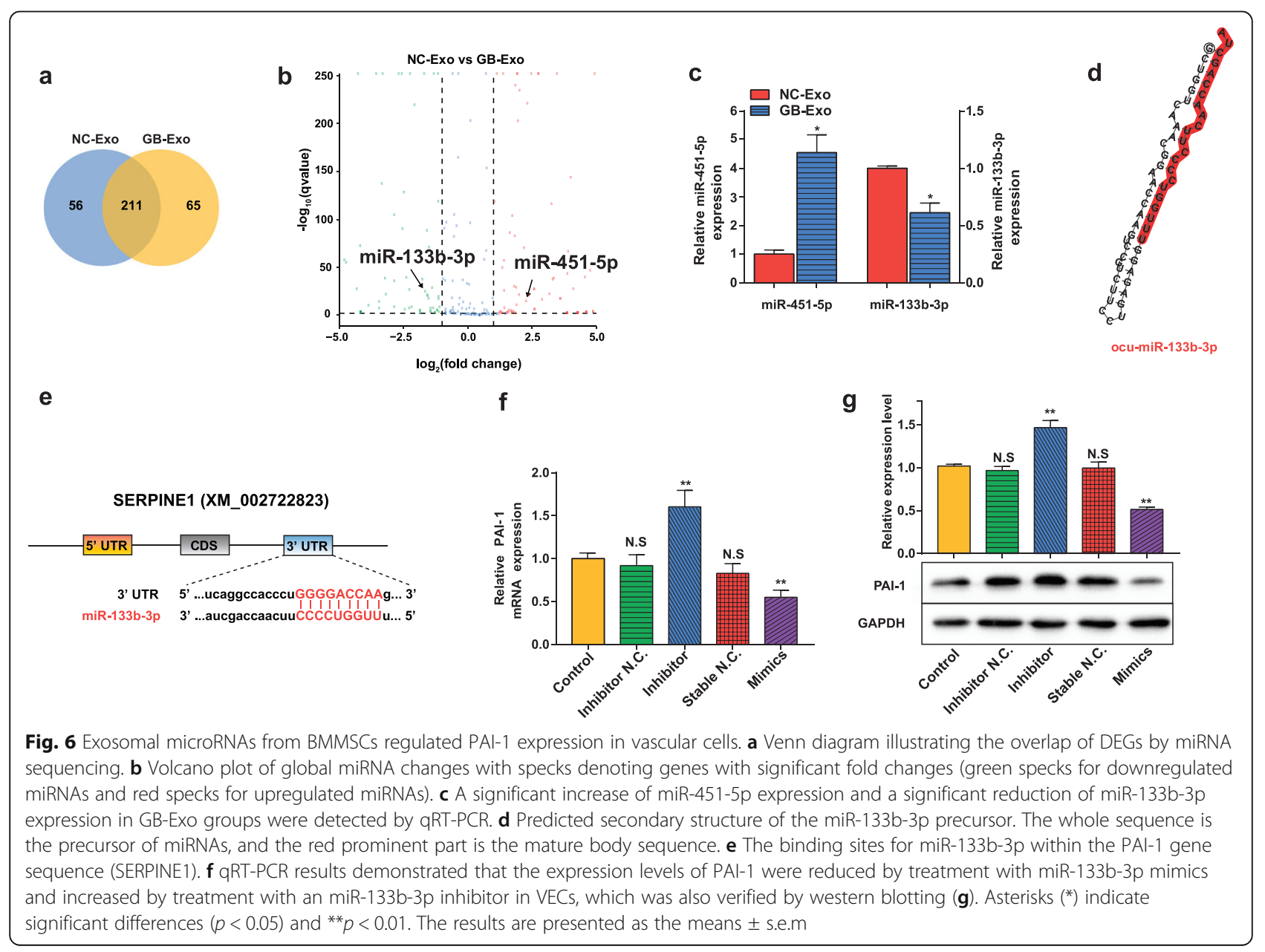


in vivo, we evaluated the pattern of PAI-1 expression from liver, femoral head bone, and peripheral blood samples by qRT-PCR (Fig. 7a) and ELISA (Fig. 7b). Firstly, the results demonstrated that miR-451-5p could directly upregulated PAI-1 expression in vivo, the relative expression of PAI-1 in miR-451-5p group revealed significantly higher levels compared to the control group in all tested tissue samples $(p<0.01)$ (Fig. 7 a and b).

Meanwhile, to investigate the preventive effects of miR-133b-3p on GC-induced ONFH, the rabbit model of ONFH was induced by intramuscularly injection of glucocorticoid, followed by intravenously administration of AgomiR 133b-3p or an equal volume saline. As exhibited in Fig. 7a and b, miR-133b-3p exerted the therapeutic function of downregulating the disorganized PAI-1 overexpression. The results showed that PAI-1 expression in miR-133b-3p group was dramatically decreased than that in the GC group $(p<0.01)$.

In histological examinations, the femoral head IHC results (Fig. 7c) indicated that the miR-451-5p group possessed much more PAI-1 positive areas than the control group, and the PAI-1 negative area of the miR-133b-3p group was clearly larger than that of the GC group.
These trends were in accordance with the molecular test results. To further evaluate the indirect effects of miR451-5p and miR-133b-3p on ONFH progression, the trabecular lacunae measured by HE staining analysis. The apoptosis of osteocytes led to the appearance of more empty lacunae. In Fig. $7 d$, it can be observed the most of lacunae were filled with osteocytes in the control group. However, there were much more empty lacunae and obviously condensed nuclei in osteocytes were found in miR-451-5p group. Additionally, compared with the GC group, the percentage of empty lacunae had decreased significantly $(p<0.01)$ with AgomiR 133b-3p injection. These results confirmed our in vitro hypothesis, they indicated that inhibition of miR-451 and miR-133 can remarkably affect the progression of GC-induced ONFH by regulating PAI-1 expression level in local microenvironment.

\section{Discussion}

Hypofibrinolysis (reduced ability to lyse thrombi) mediated by PAI-1 has been reported as a considerable pathogenic factor in NONFH. PAI-1 can combine with and inactivate tissue-type plasminogen activator (TPA)

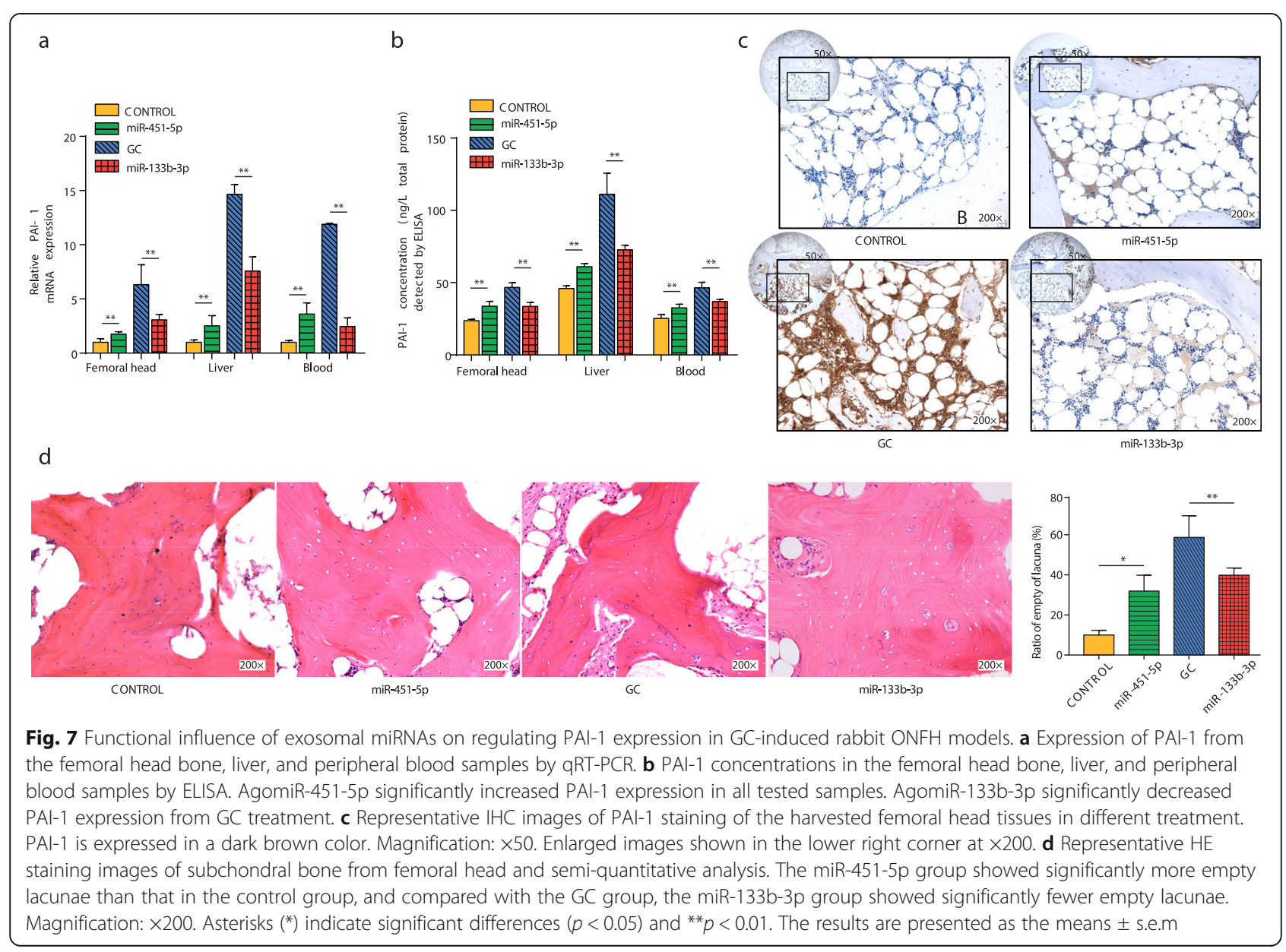


and urokinase-type plasminogen activator (UPA), and inhibit the conversion of plasminogen to plasmin, which can degrade fibrin in thrombosis. Thrombophilia and hypofibrinolysis are the main risk factors in blood supply impediment, which induces impaired circulation to femoral head. Our current study presented exploratory in vivo evidence to support a previously unrecognized "idiopathic site" role suspected for PAI-1. We reasoned that local fibrinolytic dysfunction might explain why the hip joint is the region that is most frequently affected by osteonecrosis. Utilizing a widely used rabbit GC-induced ONFH model, our observation was that the local femoral head had the most prominent response to GC stimulation. Although GC leads to an increase in systemic PAI-1 levels, which is dissimilar from other weight-bearing joints, the exceptionally high PAI-1 expression in the femoral head directly leads to alternations in fibrinolysis in that local area. Furthermore, remarkable GC-induced increases in PAI-1 were demonstrated in a variety of rabbit primarycultured cells in vitro, including BMMSCs, VSMCs, and VECs, which are the main component of the vascular structure in the femoral head. Our results further confirmed previous studies; as major fibrinolytic regulators, there is a close and vital association between PAI-1 dysregulation and GC-induced ONFH.

BMMSCs are a type of pluripotent cell. BMMSCs are particularly appealing for the treatment of ONFH. Several reports have found that BMMSC transplantation exhibited a reduced time to collapse, pain score improvements, and decreased lesion sizes with core decompressions $[35,36]$. Here, this study revealed that in our in vitro experimental conditions, short-term GC treatment has not yet driven BMMSCs to differentiate into other types of daughter cells. The effect of BMMSCs on vascular structure and tissue cells was the prominent effect. Thus, BMMSCs have played a regulatory role in vascular cells through paracrine exosomes before differentiation occurs. In addition, the role of endotoxin was investigated in a rabbit ONFH model. We found that endotoxin and glucocorticoids have a similar impact on reducing fibrinolytic capacity and can significantly increase the expression of PAI-1, but the combination did not exhibit a synergistic effect.

Utilizing exosomes derived from different stem cells has recently become a popular area of research for treatment of ONFH $[18,19,37]$. Our previous research has revealed the widespread blood supply network of the femoral head [38]. This inspired current study on intravascular coagulation and hypofibrinolysis in the femoral head. First, it is observed that GC not only reduced the fibrinolytic capacity of BMMSCs and vascular cells, but also indirectly exacerbated the loss of fibrinolytic capacity in vascular cells via BMMSC-regulating effect. Notably, this indirect influence is even greater than what was observed with direct GC treatment in vitro, especially in VECs. The same pattern was found by AlNedawi and colleagues in mast cells [22]. This phenomenon also made us realize that BMMSCs could affect the entire femoral head by virtue of the widespread vascular network. In particular, in the early stage of ONFH with large doses of GC treatment, a thinner vein would be more susceptible to the effects we observed. In addition, venous elasticity is weaker. Once a vein thrombosis was formed, it was more likely to cause changes in venous blood flow, leading to early venous stasis in ONFH.

Recent studies have revealed that stem cells could regulate the expression of specific genes in peripheral tissue cells by exosome-mediated transportation of miRNAs [39]. In this work, we screened a large number of differentially expressed miRNAs by comprehensive deep sequencing analysis of the small RNA profile from exosomes released by BMMSCs. It should be noted that miR-451-5p was found in the overexpressed miRNAs in exosomes derived from GC-treated BMMSCs. Several studies have confirmed that overexpression of miR-451 inhibited the PI3K/AKT signaling pathway by downregulated CAB39 [31, 32]. Meanwhile, the PI3K/AKT pathway was also reported to negatively regulate PAI-1 expression in vascular endothelial cells through suppressed TNF- $\alpha$ [33]. Another study suggested that a reduction in active Akt results in elevated PAI-1 expression in cancer cells [34]. Collectively, we reasoned that the overexpression of miR-451 in exosomes plays a crucial role in eventually upregulation of PAI-1 levels in vascular cells. Our in vivo experiments also supported this conclusion. Finally, an exosomal miRNA (miR133b-3p) from BMMSCs was discovered and its regulatory effect on PAI-1 was demonstrated. miR-133b-3p can target the 3'UTR of PAI-1 mRNA to reduce the expression of PAI-1. Based on our in vitro and in vivo results, we believe that engineered exosomal miR-133b-3p applied to the bone marrow of the femoral head might be a potentially efficient therapeutic approach for GC-induced ONFH.

\section{Conclusion}

In summary, our results demonstrated characteristically elevated PAI-1 levels and a procoagulant state in GCinduced rabbit ONFH model. Significantly dysregulated PAI-1 in the femoral head region might explain the susceptibility to idiopathic ONFH. This study provided considerable evidence for BMMSC exosomal miR-mediated upregulation of the fibrinolytic regulators PAI-1 in vascular cells. Moreover, future mechanistic studies of exosome-mediated BMMSC modulatory secretion will provide important insights into how this paracrine pathway contributes to ONFH physiology and pathology. Our study provides a new perspective on the etiology of NONFH. 


\section{Supplementary Information}

The online version contains supplementary material available at https://doi. org/10.1186/s13287-020-01991-2.

Additional file 1: Supplementary data. Characterization of rabbit cells. MicroRNA sequencing. Western blotting. Table S1. Primers used for RTqPCR. Table S2. Synthesized miR-133b-3p mimics and inhibitors.

\section{Abbreviations}

ONFH: Osteonecrosis of the femoral head; PAl-1: Plasminogen activator inhibitor 1; GC: Glucocorticoid; BMMSCs: Bone marrow mesenchymal stem cells; 3'UTR: 3'-Untranslated region; GB-Exo: GC-treated BMMSC exosome; NCExo: Normal control BMMSC exosomes; ALP: Alkaline phosphatase; IHC: Immunohistochemistry; VECs: Vascular endothelial cells; VSMC: Vascular smooth muscle cells; TPA: Tissue-type plasminogen activator;

UPA: Urokinase-type plasminogen activator; DEGseq: Differentially expressed gene sequencing; TEM: Transmission electron microscopy; NTA: Nanoparticle tracking analysis; DLS: Dynamic light scattering; ELISA: Enzyme-linked immunosorbent assay; qRT-PCR: Quantitative real-time polymerase chain reaction

\section{Acknowledgements}

Not applicable.

\section{Authors' contributions}

DZ and LL conceived the overall experimental design. $L L$ and $Y W$ completed most of the experiments and wrote the paper. $X Y, Y B, L A$, and $W Y$ participated in discussion of results and design of some experiments. $X W, B L$, $J L, X Z$, and $G L$ participated in analyzing data and extensively reviewed and revised the paper. JY, YX, and FC performed the histological analyses on the bone tissue fixation, section, and immunohistochemistry experiments. All authors provided intellectual input and edited and approved the final manuscript.

\section{Funding}

This work was funded by the China Postdoctoral Science Foundation (No: 194011) and Affiliated Zhongshan Hospital of Dalian University doctorinitiated special fund (No: 20172QL010).

\section{Availability of data and materials}

All data generated and/or analyzed in this study are included in this published article (and its supplementary information files).

\section{Ethics approval and consent to participate}

All experimental procedures in this study were approved and performed in accordance with the guidelines of the Animal Ethics Committee of Affiliated Zhongshan Hospital of Dalian University.

\section{Consent for publication}

Not applicable.

\section{Competing interests}

The authors declare that they have no competing interests.

\section{Author details}

${ }^{1}$ National-Local Joint Engineering Laboratory for the Development of Orthopedic Implant Materials, Affiliated Zhongshan Hospital of Dalian University, Dalian, Liaoning, People's Republic of China. 'Department of Orthopedics, Affiliated Zhongshan Hospital of Dalian University, Dalian, Liaoning, People's Republic of China. ${ }^{3}$ Medical College of Dalian University, Dalian, Liaoning, People's Republic of China. ${ }^{4}$ School of Bioengineering, Dalian University of Technology, Dalian, Liaoning, People's Republic of China. ${ }^{5}$ Department of Pathology, Affiliated Zhongshan Hospital of Dalian University, Dalian, Liaoning, People's Republic of China.
Received: 9 February 2020 Accepted: 21 October 2020

Published online: 11 November 2020

\section{References}

1. Yoon BH, Jones LC, Chen CH, Cheng EY, Cui Q, Drescher W, Fukushima W, Gangji V, Goodman SB, Ha YC, et al. Etiologic classification criteria of ARCO on femoral head osteonecrosis part 1: glucocorticoid-associated osteonecrosis. J Arthroplast. 2019;34(1):163-8 e161.

2. Lamb JN, Holton C, O'Connor P, Giannoudis PV. Avascular necrosis of the hip. BMJ. 2019;365:12178.

3. Huang G, Zhao G, Xia J, Wei Y, Chen F, Chen J, Shi J. FGF2 and FAM201A affect the development of osteonecrosis of the femoral head after femoral neck fracture. Gene. 2018:652:39-47.

4. Ghosh AK, Vaughan DE. PAl-1 in tissue fibrosis. J Cell Physiol. 2012;227(2): 493-507.

5. Rabieian R, Boshtam M, Zareei M, Kouhpayeh S, Masoudifar A, Mirzaei H. Plasminogen activator inhibitor type-1 as a regulator of fibrosis. J Cell Biochem. 2018;119(1):17-27.

6. Erickson LA, Fici GJ, Lund JE, Boyle TP, Polites HG, Marotti KR. Development of venous occlusions in mice transgenic for the plasminogen activator inhibitor-1 gene. Nature. 1990;346(6279):74-6.

7. Meltzer ME, Lisman T, Groot PG d, Meijers JC, Cessie S I, Doggen CJ, Rosendaal FR. Venous thrombosis risk associated with plasma hypofibrinolysis is explained by elevated plasma levels of TAFI and PAI-1. Blood. 2010;116(1):113-21.

8. Glueck CJ, Freiberg RA, Fontaine RN, Tracy T, Wang P. Hypofibrinolysis, thrombophilia, osteonecrosis. Clin Orthop Relat Res. 2001;386:19-33.

9. Ferrari P, Schroeder V, Anderson S, Kocovic L, Vogt B, Schiesser D, Marti HP, Ganz R, Frey FJ, Kohler HP. Association of plasminogen activator inhibitor-1 genotype with avascular osteonecrosis in steroid-treated renal allograft recipients. Transplantation. 2002;74(8):1147-52.

10. Yasar Yildiz S, Kuru P, Toksoy Oner E, Agirbasli M. Functional stability of plasminogen activator inhibitor-1. ScientificWorldJ. 2014;2014:858293.

11. Bond J, Adams S, Richards S, Vora A, Mitchell C, Goulden N. Polymorphism in the PAI-1 (SERPINE1) gene and the risk of osteonecrosis in children with acute lymphoblastic leukemia. Blood. 2011;118(9):2632-3.

12. Tan X, Cai D, Wu Y, Liu B, Rong L, Chen Z, Zhao Q. Comparative analysis of serum proteomes: discovery of proteins associated with osteonecrosis of the femoral head. Transl Res. 2006;148(3):114-9.

13. Yan Z, Hang D, Guo C, Chen Z. Fate of mesenchymal stem cells transplanted to osteonecrosis of femoral head. J Orthop Res. 2009;27(4):442-6.

14. Lebouvier A, Poignard A, Cavet M, Amiaud J, Leotot J, Hernigou P, Rahmouni A, Bierling P, Layrolle P, Rouard H, et al. Development of a simple procedure for the treatment of femoral head osteonecrosis with intraosseous injection of bone marrow mesenchymal stromal cells: study of their biodistribution in the early time points after injection. Stem Cell Res Ther. 2015;6:68.

15. Baglio SR, Rooijers K, Koppers-Lalic D, Verweij FJ, Perez Lanzon M, Zini N, Naaijkens B, Perut F, Niessen HW, Baldini N, et al. Human bone marrow- and adipose-mesenchymal stem cells secrete exosomes enriched in distinctive miRNA and tRNA species. Stem Cell Res Ther. 2015;6:127.

16. Marote A, Teixeira FG, Mendes-Pinheiro B, Salgado AJ. MSCs-derived exosomes: cell-secreted nanovesicles with regenerative potential. Front Pharmacol. 2016;7:231.

17. Ludwig N, Whiteside TL, Reichert TE. Challenges in exosome isolation and analysis in health and disease. Int J Mol Sci. 2019;20(19):4684.

18. Tao SC, Yuan T, Rui BY, Zhu ZZ, Guo SC, Zhang CQ. Exosomes derived from human platelet-rich plasma prevent apoptosis induced by glucocorticoidassociated endoplasmic reticulum stress in rat osteonecrosis of the femoral head via the Akt/Bad/Bcl-2 signal pathway. Theranostics. 2017;7(3):733-50.

19. Liu XL, Li Q, Niu X, Hu B, Chen SB, Song WQ, Ding J, Zhang CQ, Wang Y. Exosomes Secreted from Human-Induced Pluripotent Stem Cell-Derived Mesenchymal Stem Cells Prevent Osteonecrosis of the Femoral Head by Promoting Angiogenesis. Int J Biol Sci. 2017;13(2):232-44.

20. Li M, Zeringer E, Barta T, Schageman J, Cheng A, Vlassov A. Analysis of the RNA content of the exosomes derived from blood serum and urine and its potential as biomarkers. Philosophical transactions of the Royal Society of London. B Biol Sci. 2013;369(1652):20130502.

21. Hoffman EA, Perkumas KM, Highstrom LM, Stamer WD. Regulation of myocilin-associated exosome release from human trabecular meshwork cells. Invest Ophthalmol Vis Sci. 2009;50(3):1313-8. 
22. Al-Nedawi K, Szemraj J, Cierniewski CS. Mast cell-derived exosomes activate endothelial cells to secrete plasminogen activator inhibitor type 1. Arterioscler Thromb Vasc Biol. 2005;25(8):1744-9.

23. Wang WT, Li ZI, Feng J. The potential role of exosomes in the diagnosis and therapy of ischemic diseases. Cytotherapy. 2018;20(10):1204-19.

24. Qin L, Zhang G, Sheng H, Yeung K, Yeung H, Chan C, Cheung W, Griffith J, Chiu K, Leung K. Multiple bioimaging modalities in evaluation of an experimental osteonecrosis induced by a combination of lipopolysaccharide and methylprednisolone. Bone. 2006;39(4):863-71.

25. Cao F, Liu G, Wang W, Wang B, Wei X, Lu F, Yang F, Kang K, Wang Y, Yang $J$, et al. Combined treatment with an anticoagulant and a vasodilator prevents steroid-associated osteonecrosis of rabbit femoral heads by improving hypercoagulability. Biomed Res Int. 2017;2017:1624074.

26. Qin L, Zhang G, Sheng H, Yeung KW, Yeung HY, Chan CW, Cheung WH, Griffith J, Chui KH, Leung KS. Multiple bioimaging modalities in evaluation of an experimental osteonecrosis induced by a combination of lipopolysaccharide and methylprednisolone. Bone. 2006;39(4):863-71.

27. Peister A, Mellad JA, Larson BL, Hall BM, Gibson LF, Prockop DJ. Adult stem cells from bone marrow (MSCs) isolated from different strains of inbred mice vary in surface epitopes, rates of proliferation, and differentiation potential. Blood. 2004;103(5):1662-8.

28. Lombardi DM, Reidy MA, Schwartz SM. Methodologic considerations important in the accurate quantitation of aortic smooth muscle cell replication in the normal rat. Am J Pathol. 1991;138(2):441-6.

29. Cybulsky Ml, Gimbrone MA Jr. Endothelial expression of a mononuclear leukocyte adhesion molecule during atherogenesis. Science. 1991;251(4995): 788-91

30. Ying Y, Li J, Xie H, Yan H, Jin K, He L, Ma X, Wu J, Xu X, Fang J et al: CCND1, NOP14 and DNMT3B are involved in miR-502-5p-mediated inhibition of cell migration and proliferation in bladder cancer. Cell Prolif. 2020;53(2):e12751.

31. Tian Y, Nan Y, Han L, Zhang A, Wang G, Jia Z, Hao J, Pu P, Zhong Y, Kang C. MicroRNA miR-451 downregulates the PI3K/AKT pathway through CAB39 in human glioma. Int J Oncol. 2012;40(4):1105-12.

32. Hur W, Lee JH, Kim SW, Kim J-H, Bae SH, Kim M, Hwang D, Kim YS, Park T, Um S-J. Downregulation of microRNA-451 in non-alcoholic steatohepatitis inhibits fatty acid-induced proinflammatory cytokine production through the AMPK/AKT pathway. Int J Biochem Cell Biol. 2015:64:265-76.

33. Mukai Y, Wang C-Y, Rikitake Y, Liao JK. Phosphatidylinositol 3-kinase/protein kinase Akt negatively regulates plasminogen activator inhibitor type 1 expression in vascular endothelial cells. Am J Phys Heart Circ Phys. 2007; 292(4):H1937-42.

34. Whitley BR, Beaulieu LM, Carter JC, Church FC. Phosphatidylinositol 3-kinase/ Akt regulates the balance between plasminogen activator inhibitor- 1 and urokinase to promote migration of SKOV-3 ovarian cancer cells. Gynecol Oncol. 2007;104(2):470-9.

35. Li R, Lin QX, Liang XZ, Liu GB, Tang H, Wang Y, Lu SB, Peng J. Stem cell therapy for treating osteonecrosis of the femoral head: from clinical applications to related basic research. Stem Cell Res Ther. 2018;9(1):291.

36. Zhao D, Cui D, Wang B, Tian F, Guo L, Yang L, Liu B, Yu X. Treatment of early stage osteonecrosis of the femoral head with autologous implantation of bone marrow-derived and cultured mesenchymal stem cells. Bone. 2012; 50(1):325-30.

37. Liao W, Ning Y, Xu HJ, Zou WZ, Hu J, Liu XZ, Yang Y, Li ZH. BMSC-derived exosomes carrying microRNA-122-5p promote proliferation of osteoblasts in osteonecrosis of the femoral head. Clin Sci (Lond). 2019;133(18):1955-75.

38. Zhao D, Qiu X, Wang B, Wang Z, Wang W, Ouyang J, Silva RM, Shi X, Kang $\mathrm{K}, \mathrm{Xu}$ D. Epiphyseal arterial network and inferior retinacular artery seem critical to femoral head perfusion in adults with femoral neck fractures. Clin Orthop Related Res. 2017:475(8):2011-23.

39. Xu R, Shen X, Si Y, Fu Y, Zhu W, Xiao T, Fu Z, Zhang P, Cheng J, Jiang H. Micro RNA-31a-5p from aging BMSC $s$ links bone formation and resorption in the aged bone marrow microenvironment. Aging Cell. 2018;17(4):e12794.

\section{Publisher's Note}

Springer Nature remains neutral with regard to jurisdictional claims in published maps and institutional affiliations.

\section{Ready to submit your research? Choose BMC and benefit from:}

- fast, convenient online submission

- thorough peer review by experienced researchers in your field

- rapid publication on acceptance

- support for research data, including large and complex data types

- gold Open Access which fosters wider collaboration and increased citations

- maximum visibility for your research: over $100 \mathrm{M}$ website views per year

At BMC, research is always in progress.

Learn more biomedcentral.com/submissions 